Studies in Perception and Action III

B. G. Bardy, R. J. Bootsma, and Y. Guiard (Eds.)

(C) 1995 Lawrence Erlbaum Associates, Inc.

\title{
Haptic Surface Aftereffect is of Central, Not Peripheral Origin
}

\author{
Ingrid M. L. C. Vogels, Astrid M. L. Kappers, and Jan J. Koenderink
}

Helmholtz Institute, University of Utrecht, The Netherlands

In haptics, a space sense that relies solely on touch, the perceived flatness of a surface (phenomenal flatness) can be strongly influenced by a previously touched curved surface. The sign of the geometrical curvature of an object does not necessarily correspond to the concave-convex judgments of a human person. This is perhaps surprising because most of us tend to assume that we need only put our hand on an object to obtain a veridical impression of its shape. We have shown (Vogels, Kappers, \& Koenderink, 1994) that phenomenal flatness is shifted towards the curvature of a previously touched surface (conditioning surface). This novel aftereffect occurs almost immediately: After only $2 \mathrm{~s}$ of touching the conditioning surface, the aftereffect is already evident. It is also a strong effect: After a conditioning period of $5 \mathrm{~s}$, a sphere with radius $1 \mathrm{~m}$ may be judged to be llat.

The experiment reported here was designed to gain insight into the processes involved in the haptic surface aftereffect. Skin, joint and muscle receptors could be involved, since it is known that these receptors adapt. Muscle receptors, however, adapt very slowly. Higher centres of the brain might also play a role. We studied the decay of the aftereffect by varying the time interval between the conditioning period and the touching of the test surface.

Three conditions were investigated, which differed mainly in the degree of stimulation of skin and joint receptors during the interval.

In the passive condition, participants held their hand in the air in a comfortable manner. No pressure was applied to the skin and the joints were in a mid-position (joint receptors respond only near the extreme positions of the joints). Because the skin and joint receptors were minimally stimulated, we could expect either that the adaptation would be maintained during the interval or that the teceptors would recover from adaptation.

In the fist condition, the participant's lingers were in contact with his/her own skin, with the joints in an extreme position. This situation is reminiscent of touching a strongly curved object such as a pencil, which might cause the receptors to adapt to positive curvature.

In the dynamic condition, purticipants suecessively bent and stretched their fingers. The skin and joint receptors were stimulated but could not adapt to pressure or position because of the alternating stimulation. Movements of the fingers were supposed to cause the receptors to recover tapidly. 


\section{Method}

So far two naive right-handers (as defined by Coren, 1993) participated in the experiment. The objects we used had a spherically curved upper surface, a flat bottom and a diameter of $20 \mathrm{~cm}$ (see Figure 1A). Object curvature (expressed as reciprocal radius - a sphere with radius $2 \mathrm{~m}$ has a curvature of $0.5 \mathrm{~m}^{-1}-$, and with positively and negatively curved surfaces referred to as convex and concave, respectively) ranged from $-4 \mathrm{~m}^{-1}$ to $4 \mathrm{~m}^{-1}$. Participants first placed their right hand on a conditioning surface. After $10 \mathrm{~s}$, they removed their hand and, after a certain time interval, put it on a second, test surface. Participants had to decide whether this test surface was convex or concave. No exploratory movements were allowed.

Three conditioning surfaces were used: $-4 \mathrm{~m}^{-1}, 0 \mathrm{~m}^{-1}$ and $4 \mathrm{~m}^{-1}$. In the passive condition the interval was $5,20,40$, or $80 \mathrm{~s}$; in the fist condition 5,20 , or $40 \mathrm{~s}$; in the dynamic condition 5 or $10 \mathrm{~s}$. For each combination of interval duration and condition, the conditioning surfaces were presented 15 times together with 9 differently curved test surfaces. A psychometric curve was fitted to the percentage convex judgments (see Figure 1B).

\section{Results}

Both participants showed similar results. Figure $1 \mathrm{~B}$ presents an example of a psychometric curve in the case of a flat conditioning surface, an interval of $20 \mathrm{~s}$, and the passive condition. Hunter (1954) already reported that even without prior conditioning, haptic

A

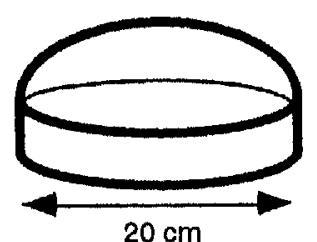

Figure 1. Pancl A: An example of a convex spherically slaped curved stimulus. Panel $B:$ The percentage of convex judgments of Participant MV for a flat conditioning surface, an interval of $20 \mathrm{~s}$, and the passive condition. 


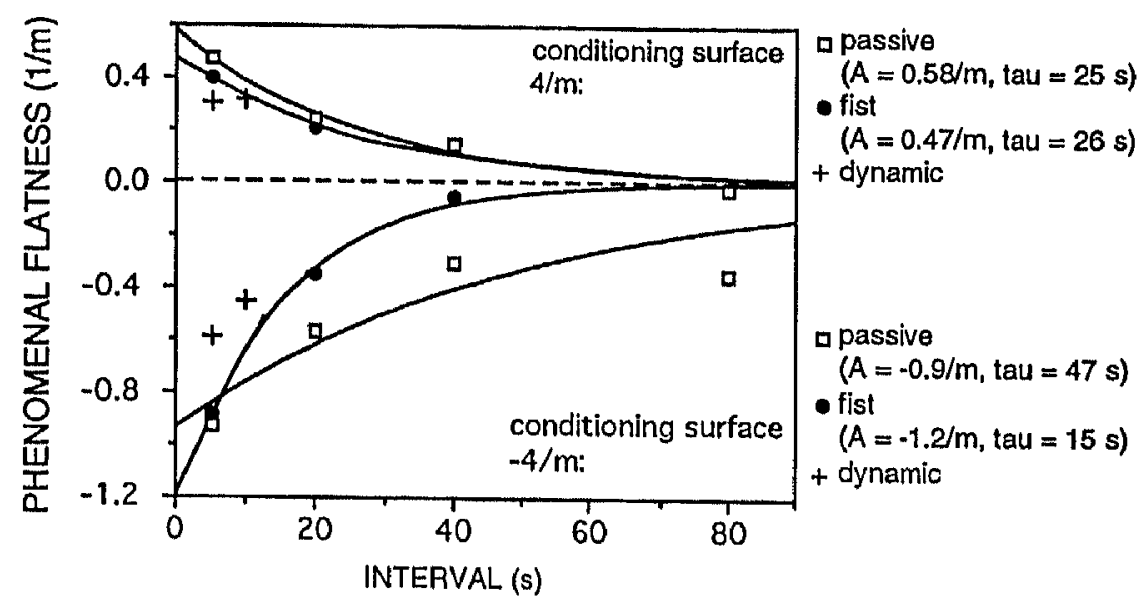

Figure 2. The relation between length of the interval and phenomenal flatness. Because of small day-to-day variations in phenomenal flatness, the shift induced by a flat conditioning surface has been subtracted from the shift induced by a curved conditioning surface.

perception is not veridical. He found that in general strips that were perceived as straight were typically curved. In Figure 1B the shift of the $50 \%$ point indicates a similar effect. For the flat conditioning surface, we found that this shift did not depend on either the length of the interval or the condition. The average shift was $-0.54 \pm 0.03 \mathrm{~m}^{-1}$. For the curved conditioning surfaces, in contrast, both the length of the interval and the condition did influence the shift, as shown in Figure 2. Increasing the length of the interval resulted in a decay of the aftereffect. For the passive and active conditions an exponential decay function was fitted: $P(t)=A \mathrm{e}^{-v / \tau}$. The two time constants were significantly different in the case of the concave conditioning surface, but not in the case of the convex conditioning surface. The decay of the aftereffect in the dynamic conditions was somewhat larger than in the other conditions.

Under all circumstances, the threshold (inversely proportional to the slope of the psychometric curve) was $0.21 \pm 0.02 \mathrm{~m}^{-1}$.

\section{Discussion}

Irrespective of the interval condition, the decay of the aftereffect was 10 to 20 times slower than the build-up, which was very fast $(\tau=2 s)$. So louching a curved object for a short time has a noticeable influence on the judged curvature of another object after a passive period of about $40 \mathrm{~s}$ and even after an active period of about $20 \mathrm{~s}$.

There was a difference in the decay of the aftereflect under different conditions. However, if the skin and joint receptors play a major role in the aftereffect, one would expect the differences to be larger (sce the introductory section). In the fist condition the phenomenal flatness was not shifted in the same direction for all conditioning 
surfaces, so apparently no new adaptation occurred. In the dynamic condition, no rapid decay of the adaptation took place.

The above data do not support the hypothesis that the aftereffect is due to adaptation of skin and joint receptors (peripheral origin). It follows that the aftereffect is probably of central origin, although more psychophysical and physiological experiments are needed to confirm this.

Acknowledgments. This research was supported by the Netherlands Organization of Scientific Research (NWO).

\section{References}

Coren, S. (1993). The left-hander syndrome. New York: Vintage Books.

Hunter, I.(1954). Tactile-kinaesthetic perception of straighteness in blind and sighted humans. Quarterly Journal of Experimental Psychology, 6, 149-154.

Vogels, I. M. L. C., Kappers, A. M. L., \& Koenderink, J. J. (1994). Flat surfaces often feel curved. Technical Report of the Utrecht Biophysics Research Institute, FM-026, Utrecht, The Netherlands. 